\title{
Fabrication of ZnSe quantum dots under Volmer-Weber mode by metalorganic chemical vapor deposition
}

\author{
M. C. Harris Liao, Y. H. Chang, ${ }^{\text {a) }}$ Y. F. Chen, and J. W. Hsu \\ Department of Physics, National Taiwan University, Taipei 106, Taiwan, Republic of China \\ J. M. Lin and W. C. Chou \\ Department of Physics, Chung-Yuan Christian University, Chung-Li 320, Taiwan, Republic of China
}

(Received 18 November 1996; accepted for publication 25 February 1997)

\begin{abstract}
The possibility of fabricating quantum dots under Volmer-Weber growth mode is investigated. Layers of $\mathrm{ZnSe} / \mathrm{ZnS}$ were grown by metalorganic chemical vapor deposition on both $\mathrm{Si}$ and $\mathrm{GaAs}$ substrates. The images of surface morphology, taken by atomic force microscopy, showed that the layers were grown in three-dimensional islands. Blueshift was observed in the photoluminescence spectra up to room temperature for these samples. This blueshift was shown to originate from the $\mathrm{ZnSe}$ islands. The effect of $\mathrm{ZnSe}$ growth duration was investigated. It was found that the blueshift increased with shorter $\mathrm{ZnSe}$ growth durations, but this near band gap emission disappeared when the $\mathrm{ZnSe}$ growth duration was longer than $5 \mathrm{~s}$. Effects of quantum confinement and strain were considered to resolve the origin of the blueshift. Our result suggests that carrier confinement plays a dominant role. () 1997 American Institute of Physics. [S0003-6951(97)00917-0]
\end{abstract}

II-VI wide band gap semiconductors, especially ZnSe, have been long studied for their potential in the applications of optoelectronic devices. Laser diodes based on $\mathrm{ZnSe}$ were successfully demonstrated to operate under $\mathrm{cw}$ mode at room temperature. ${ }^{1,2}$ To the best of our knowledge, the longest lifetime reported was $100 \mathrm{~h}$. How to prolong the lifetime remains a key challenge. Both the $p$-type contacting problem and the high defect density hinder this job. ${ }^{3-6}$ Even though $p$-type doping levels have reached $10^{18} \mathrm{~cm}^{-3}$, this appears to be still too low to produce low resistance contacts to $p$-doped layers. On the other hand, defects are generated at the II-VI/GaAs interface due to heterovalent nucleation, although lattice matching can be maintained by ternary or quarternary alloys. Two-dimensional (2D) growth often does not proceed at the interface. Defects are formed when threedimensional (3D) growth takes place. Unlike their competitor, the nitride material system, ZnSe-based diodes were heavily degraded by these defects.

Meanwhile, quantum dots have brought much attention for their potential to increase electron-hole recombination efficiency. Such dots are effectively 0D. They have higher quantum efficiency, compared to 2D quantum wells and 1D quantum wires. ${ }^{7}$ Various ways of producing quantum dots were demonstrated. Self-organized quantum dots have become most attractive because of their nondestructive and nongrowth interrupted nature. The dots were epitaxially formed on highly mismatched substrates. ${ }^{8-11}$ They were found to grow under the Stranski-Krastanov mode: a layer was first grown in 2D, and 3D islands were formed after some critical thickness was reached. These dots were introduced to accommodate the strain energy without creating dislocations between the dots and the underlying layer. These dots are epitaxially and coherently grown. The term "self-assembled" is used to refer to these kinds of dots. Recently, laser structures have been demonstrated on such self-organized quantum dot systems. ${ }^{12}$ But by far, research

${ }^{a)}$ Electronic mail: yhchang@phys.ntu.edu.tw on self-organized quantum dots was mostly done in systems such as $\mathrm{SiGe}$, InAs, and other III-V compounds. Few reports of II-VI self-organized dots could be found ${ }^{13}$ and most of the self-organized quantum dots were prepared by molecular beam epitaxy (MBE). This might indicate that the selforganized quantum dots need precise control of the layer-bylayer growth, as well as the layer thickness which is typically within several monolayers. This task is more easily done on a MBE system equipped with a reflection high-energy electron diffraction (RHEED) monitor.

In this letter, we present a very simple way to produce ZnSe quantum dots by metalorganic chemical vapor deposition (MOCVD). It is known that there are three modes of epitaxial growth. They are:

(1) Frank-van der Merwe mode, a layer-by-layer growth;

(2) Volmer-Weber mode, an island growth;

(3) Stranski-Krastanow mode, a layer-by-layer plus island growth.

Which growth mode is favored during epitaxial growth depends on the interfacial energy and the degree of lattice mismatch. We demonstrate here that quantum dots could also be grown under the Volmer-Weber mode, instead of the Stranski-Krastanow mode. The basic idea is to embed quantum dots directly in another material with wider band gap, instead of introducing a 2D layer. To do so, a rough layer resulted from island growth was first prepared as a starting surface. Quantum dots were then grown on this rough surface. Dot size was controlled by growth duration. Various degrees of blueshift in photoluminescence (PL) spectra were observed with respect to different growth durations. Atomic force microscopy (AFM) was also used to observe surface morphology. The PL spectra were obtained by pumping samples with a $\mathrm{He}-\mathrm{Cd}$ laser at an average power density of $50 \mathrm{MW} / \mathrm{cm}^{2}$. A $0.85 \mathrm{~m}$ monochromator, a photomultiplier tube, and standard lock-in technique were used to detect a signal. The AFM images were taken under constant high mode on an AFM model CP from Park Scientific Instruments. Ultralever ${ }^{\mathrm{TM}}$ tips of $0.6 \mu \mathrm{m}$ thickness were used. 


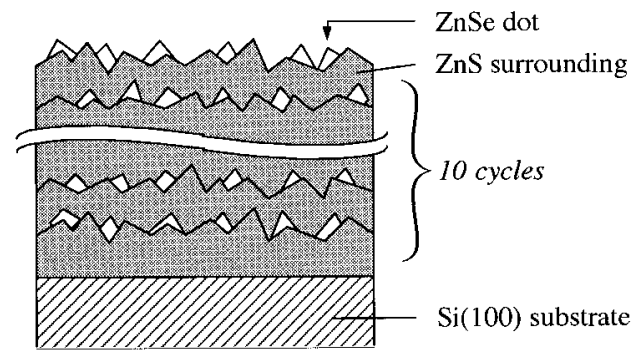

FIG. 1. Schematic of sample structure on Si. Ten cycles of the $\mathrm{ZnSe} / \mathrm{ZnS}$ structure were repeated. The growth duration of $\mathrm{ZnSe}$ ranged from 1 to $20 \mathrm{~s}$ while the growth duration of $\mathrm{ZnS}$ was $40 \mathrm{~s}$. The growth rates were calculated to be about $10 \AA / \mathrm{s}$ and $100 \AA / \mathrm{min}$ for $\mathrm{ZnSe}$ and $\mathrm{ZnS}$, respectively. For samples grown on GaAs, a thicker ZnS layer was first grown to release the strain, then the ten cycles of $\mathrm{ZnSe} / \mathrm{ZnS}$ were grown.

The samples were grown by MOCVD. Dimethylzinc (DMZn), diethylselenide (DESe), and diethylsulfide (DES) were used as precursors. Typical flow rates were 10, 30, and $30 \mu \mathrm{mol} / \mathrm{min}$, respectively. The growth pressure was kept at 760 Torr and the growth temperature was $530{ }^{\circ} \mathrm{C} .1500 \mathrm{sccm}$ $\mathrm{H}_{2}$ was fed into the growth chamber. $\mathrm{GaAs}(001)$ and $\mathrm{Si}(001)$ were both used as substrates. Figure 1 shows the sample structure. Basically, $\mathrm{ZnSe}$ was introduced to form quantum dots while $\mathrm{ZnS}$ was used to separate each $\mathrm{ZnSe}$ layer. Ten cycles of such $\mathrm{ZnSe} / \mathrm{ZnS}$ structure were repeated. The growth duration of $\mathrm{ZnSe}$ in each cycle was varied from 1 to $20 \mathrm{~s}$ while that of $\mathrm{ZnS}$ was fixed at $40 \mathrm{~s}$. The growth rates were calculated to be about $10 \AA / \mathrm{s}$ and $100 \AA / \mathrm{min}$ for $\mathrm{ZnSe}$ and $\mathrm{ZnS}$ in this growth condition, respectively. For layers grown on $\mathrm{GaAs}(001), 4 \mathrm{H}_{2} \mathrm{SO}_{4}: \mathrm{H}_{2} \mathrm{O}_{2}: \mathrm{H}_{2} \mathrm{O}$ and $\mathrm{HCl}$ were used to etch the substrates before loading to the growth chamber. Prior to layer growth, the substrates were heated at $550{ }^{\circ} \mathrm{C}$ in $\mathrm{H}_{2}$ ambient for $40 \mathrm{~min}$. A ZnSe buffer layer at $400{ }^{\circ} \mathrm{C}$ was first grown. It was followed by a thick $\mathrm{ZnS}$ layer to release the heteroepitaxial strain. For layers grown on $\mathrm{Si}(001)$, chemical cleaning was performed by:

(1) $4 \mathrm{H}_{2} \mathrm{SO}_{4}: \mathrm{H}_{2} \mathrm{O}, 10 \mathrm{~min}$;

(2) $\mathrm{HF}: 3 \mathrm{H}_{2} \mathrm{O}, 1$ min.

Prior to layer growth, the substrates were heated up to $900{ }^{\circ} \mathrm{C}$ in DES and $\mathrm{H}_{2}$ ambient for 10 min.

Figure 2 shows the surface morphology image of a onecycle $\mathrm{ZnSe} / \mathrm{ZnS}$ structure with $\mathrm{ZnSe}$ growth duration set on 1 $\mathrm{s}$. The behavior of $3 \mathrm{D}$ growth is obvious. Isolated islands are found. Some larger spots are also observed but they are actually the aggregation of the smaller islands. The measured island size is about $250 \AA$ A. Figure 3(a) shows a typical PL spectrum of our samples at $10 \mathrm{~K}$. The only emission around $3.1 \mathrm{eV}$ is higher than the near band gap emission of $2.8 \mathrm{eV}$ in bulk $\mathrm{ZnSe}$, but it is also within the band gap of $\mathrm{ZnS}$. To show that this emission is significant, we have to first rule out the possibility that this emission comes from the deep levels of $\mathrm{ZnS}$. Therefore, we prepared another sample in exactly the same way except that the $\mathrm{ZnSe}$ growth duration was set to zero, i.e., there was no $\mathrm{ZnSe}$ incorporated. As we can see in its spectrum shown in Fig. 3(b), there is no corresponding emission around $3.1 \mathrm{eV}$, except for some weak deep level emission around $2.4 \mathrm{eV}$. So we believe that the emission around $3.1 \mathrm{eV}$ originates from the $\mathrm{ZnSe}$ layers.


FIG. 2. AFM image of a one-cycle $\mathrm{ZnSe} / \mathrm{ZnS}$ structure. The growth duration of $\mathrm{ZnSe}$ was $1 \mathrm{~s}$ and that of $\mathrm{ZnS}$ was $40 \mathrm{~s}$. This image shows that $\mathrm{ZnSe} / \mathrm{ZnS}$ forms 3D islands. These mixed $\mathrm{ZnSe} / \mathrm{ZnS}$ islands are about 250 $\AA$ in diameter.

This emission shows a blueshift about $300 \mathrm{meV}$ from the near band gap emission in bulk $\mathrm{ZnSe}$. With the 3D growth nature in mind, we therefore assign the blueshift to the result of those small $\mathrm{ZnSe}$ islands, or dots, formed during the 3D growth. Figure 4 shows the photoluminescence spectra of different $\mathrm{ZnSe}$ growth durations while the $\mathrm{ZnS}$ spacer duration is fixed at $40 \mathrm{~s}$. Blueshift was observed for samples with short $\mathrm{ZnSe}$ growth durations (1-3 in.) only. The blueshift increases with respect to the decrease in $\mathrm{ZnSe}$ growth duration. This behavior is explained by the effect of quantum confinement. Shorter $\mathrm{ZnSe}$ growth durations result in dot size reduction. This reduction in dot size enhances quantum confinement effect, so as to increase the blueshift. In addition, the observation of room temperature PL emission, as

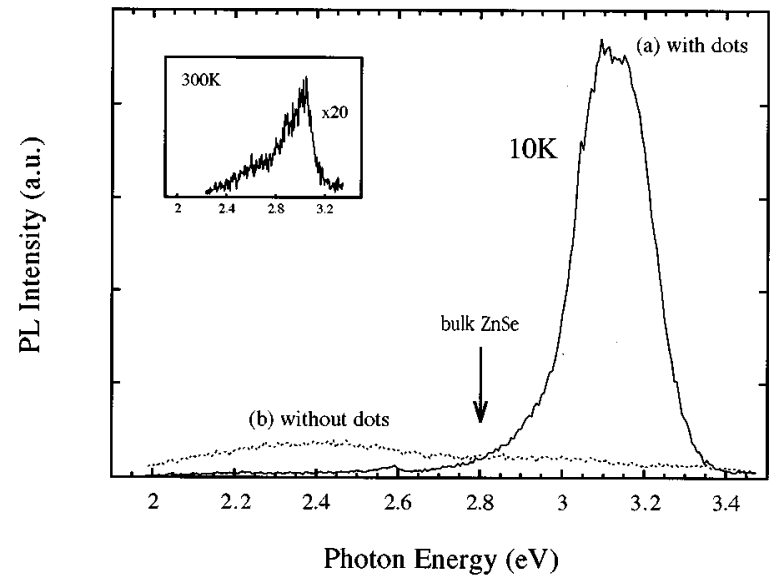

FIG. 3. (a) PL spectrum of samples with $\mathrm{ZnSe}$ duration $1 \mathrm{~s}$ and $\mathrm{ZnS}$ duration $40 \mathrm{~s}$. The emission shows a blueshift from the free exciton emissions in bulk $\mathrm{ZnSe}$, which is indicated by the arrow. The inset shows the corresponding emission at room temperature. The intensity is reduced by $1 / 20$. (b) PL spectrum of samples with no $\mathrm{ZnSe}$ incorporated. The blueshifted emission is missing in this spectrum. This suggests that the blueshifted emission results from the $\mathrm{ZnSe}$ layers. 


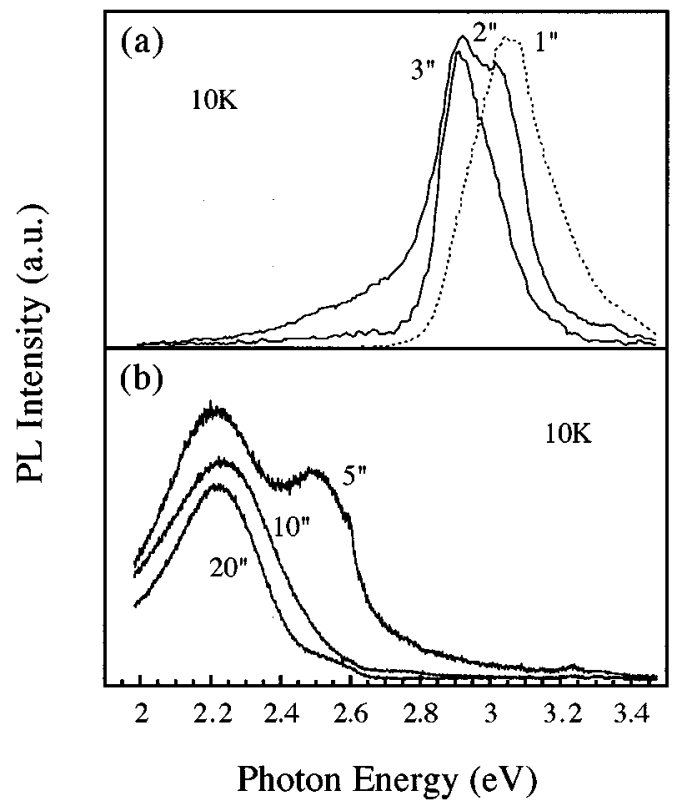

FIG. 4. PL spectra of different $\mathrm{ZnSe}$ durations. The $\mathrm{ZnS}$ growth duration is $40 \mathrm{~s}$. (a) Varied degree of blueshift is shown for 1, 2, and 3 in. samples. (b) Only deep emissions show up for 5, 10, and 20 in. samples, instead of the near band gap emissions as in (a)

shown in the inset of Fig. 3, also supports the interpretation of strong quantum confinement. However, it is reasonable to suspect that the blueshift due to carrier confinement can be as large as $300 \mathrm{meV}$ for $\mathrm{ZnSe}$ dots of which the size is $250 \AA$ in diameter. This difficulty can be resolved by adopting the model obtained for III-V quantum dots. It has been explained that the diameter of dots observed in AFM is within the base area of the dot, while the emission comes from the tip of the cone-shaped dots. For InAs dots of which the size is $250 \AA$ in diameter, the blueshift can be as large as 700 $\mathrm{meV} .{ }^{14}$ Therefore, our observation on the blueshift is within the acceptable range. For samples with long $\mathrm{ZnSe}$ growth durations (5-20 in.), only weak deep emissions appear. This is probably due to the fact that misfit dislocation is introduced to release strain in the samples where the size of the larger islands exceeds the critical thickness. As to the smaller dots where the strain is not released, blueshift could also be caused by the strain. However, the blueshift due to strain effect in the $\mathrm{ZnSe} / \mathrm{ZnS}$ system has been estimated to be about $40 \mathrm{meV},{ }^{15-18}$ which is much smaller than the blueshift observed in our measurement. Thus, we conclude that carrier confinement effect dominates the blueshift for our quantum dots.

In conclusion, controllable blueshift of PL emission, which was shown to originate from $\mathrm{ZnSe}$ layers fabricated by island growth, was observed. This blueshift was linked to the small $\mathrm{ZnSe}$ islands embedded within $\mathrm{ZnS}$. The PL emission was found to persist up to room temperature. The feature that the blueshift is enhanced by shorter $\mathrm{ZnSe}$ growth durations provides strong evidence that quantum confinement effect plays a major role. Our result thus demonstrates that quantum dots can be grown under the Volmer-Weber mode. This opens a new avenue for the study and fabrication of zero-dimensional quantum structures.

This work is supported by the National Science Council of the Republic of China.

${ }^{1}$ M. A. Haase, P. F. Baude, M. S. Hafedorn, J. Qiu, J. M. DePuydt, H. Cheng, S. Guha, G. E. Hofler, and B. J. Wu, Appl. Phys. Lett. 63, 2315 (1993).

${ }^{2}$ N. Nakayama, S. Itoh, T. Ohata, K. Nakano, H. Okuyama, M. Ozawa, A. Ishbashi, M. Ikeda, and Y. Mori, Electron. Lett. 29, 1488 (1993).

${ }^{3}$ Y. Fan, J. Han, L. He, J. Saraie, R. L. Gunshor, M. Hagerott, H. Jeon, A. V. Nurmiko, G. C. Hua, and N. Otsuka, Appl. Phys. Lett. 61, 3161 (1992).

${ }^{4}$ Y. Lansari, J. Ren, B. Sneed, K. A. Bowers, J. W. Cook, Jr., and J. F. Schetzina, Appl. Phys. Lett. 61, 2554 (1992).

${ }^{5}$ L. H. Kuo, L. Salamanca-Riba, B. J. Wu, G. Hofler, J. M. DePuydt, and H. Cheng, Appl. Phys. Lett. 67, 3298 (1995).

${ }^{6}$ B. J. Wu, L. H. Kuo, J. M. DePuydt, G. M. Haugen, M. A. Haase, and L. Salamanca-Riba, Appl. Phys. Lett. 68, 379 (1996).

${ }^{7}$ Y. Arakawa and H. Sakaki, Appl. Phys. Lett. 40, 939 (1982).

${ }^{8}$ D. J. Eaglesham and M. Cerullo, Phys. Rev. Lett. 64, 1943 (1990).

${ }^{9}$ J. Tersoff, C. Teichert, and M. G. Lagally, Phys. Rev. Lett. 76, 1675 (1996)

${ }^{10}$ G. S. Solomon, J. A. Trezza, A. F. Marshall, and J. S. Harris, Jr., J. Vac. Sci. Technol. B 14, 2208 (1996).

${ }^{11}$ E. R. Glaser, B. R. Bennett, B. V. Shanabrook, and R. Magno, Appl. Phys. Lett. 68, 3614 (1996).

${ }^{12}$ H. Shoji, Y. Nakata, K. Mukai, Y. Sugiyama, M. Sugawara, N. Yokoyama, and H. Ishikawa, Electron. Lett. 32, 2023 (1996).

${ }^{13}$ Y. H. Wu, K. Arai, and T. Yao, Phys. Rev. B 53, 10485 (1996).

${ }^{14}$ J. Y. Marzin, J. M. Gerard, A. Izrael, D. Barrier, and G. Bastard, Phys. Rev. Lett. 73, 716 (1994).

${ }^{15}$ T. Taguchi, Y. Kawakami, and Y. Yamada, Physica B 191, 23 (1993).

${ }^{16}$ C. G. Van de Walle and R. M. Martin, Phys. Rev. B 35, 8154 (1987).

${ }^{17}$ K. Shahzad and D. J. Olego, Phys. Rev. B 38, 1417 (1988).

${ }^{18}$ C. Trager-Cowan, P. J. Parbrook, B. Henderson, and K. P. O'Donnell, Semicond. Sci. Technol. 7, 536 (1992). 